ARTICLE

\title{
The nature of active sites for carbon dioxide electroreduction over oxide-derived copper catalysts
}

\author{
Dongfang Cheng (10) 1,2,6, Zhi-Jian Zhao (1) 1,2,6, Gong Zhang (10) 1,2, Piaoping Yang1,2, Lulu Li,2, Hui Gao ${ }^{1,2}$, \\ Sihang Liu (10 ${ }^{1,2}$, Xin Chang ${ }^{1,2}$, Sai Chen ${ }^{1,2}$, Tuo Wang ${ }^{1,2}$, Geoffrey A. Ozin ${ }^{3}$, Zhipan Liu (1) ${ }^{4}$ \& \\ Jinlong Gong (iD $1,2,5 \bowtie$
}

The active sites for $\mathrm{CO}_{2}$ electroreduction $\left(\mathrm{CO}_{2} \mathrm{R}\right)$ to multi-carbon $\left(\mathrm{C}_{2+}\right)$ products over oxidederived copper (OD-Cu) catalysts are under long-term intense debate. This paper describes the atomic structure motifs for product-specific active sites on OD-Cu catalysts in $\mathrm{CO}_{2} \mathrm{R}$. Herein, we describe realistic OD-Cu surface models by simulating the oxide-derived process via the molecular dynamic simulation with neural network (NN) potential. After the analysis of over 150 surface sites through NN potential based high-throughput testing, coupled with density functional theory calculations, three square-like sites for $\mathrm{C}-\mathrm{C}$ coupling are identified. Among them, $\Sigma 3$ grain boundary like planar-square sites and convex-square sites are responsible for ethylene production while step-square sites, i.e. $n(111) \times(100)$, favor alcohols generation, due to the geometric effect for stabilizing acetaldehyde intermediates and destabilizing $\mathrm{Cu}-\mathrm{O}$ interactions, which are quantitatively demonstrated by combined theoretical and experimental results. This finding provides fundamental insights into the origin of activity and selectivity over Cu-based catalysts and illustrates the value of our research framework in identifying active sites for complex heterogeneous catalysts.

\footnotetext{
${ }^{1}$ Key Laboratory for Green Chemical Technology of Ministry of Education, School of Chemical Engineering and Technology, Tianjin University, 300072 Tianjin, China. ${ }^{2}$ Collaborative Innovation Center of Chemical Science and Engineering (Tianjin), 300072 Tianjin, China. ${ }^{3}$ Department of Chemistry, University of Toronto, Toronto, ON, Canada. ${ }^{4}$ Collaborative Innovation Centre of Chemistry for Energy Material, Shanghai Key Laboratory of Molecular Catalysis and Innovative Materials, Key Laboratory of Computational Physical Science, Department of Chemistry, Fudan University, Shanghai, China. ${ }^{5}$ Joint School of National University of Singapore and Tianjin University, International Campus of Tianjin University, Binhai New City, 350207 Fuzhou, China. ${ }^{6}$ These authors contributed equally: Dongfang Cheng, Zhi-Jian Zhao. ${ }^{凶}$ email: jlgong@tju.edu.cn
} 
T he excessive utilization of fossil fuels has led to dramatic anthropogenic climate change and serious energy crisis. Electrochemical carbon dioxide reduction $\left(\mathrm{CO}_{2} \mathrm{R}\right)$ to renewable fuels and feedstocks has gained significant attention, and been regarded as a promising strategy to close the carbon cycle and store renewable energy ${ }^{1-3}$. Copper is by far the most promising metal capable of converting $\mathrm{CO}_{2}$ into multi-carbon hydrocarbons and oxygenates ${ }^{4-8}$. Among the $\mathrm{Cu}$-based catalysts, oxide-derived copper $(\mathrm{OD}-\mathrm{Cu})$ is particularly attractive catalyst due to its remarkable selectivity towards potentially valuable $\mathrm{C}_{2+}$ products at relatively low overpotentials, which exhibits the prospect of large-scale industrialization ${ }^{9-11}$.

The outstanding catalytic performance for $\mathrm{C}_{2}$ products over $\mathrm{OD}-\mathrm{Cu}$ catalysts is under intense debate, which is possibly related to, for instance, subsurface oxygen ${ }^{12}$, grain boundaries ${ }^{10,13}$, exposed facet ${ }^{14}$, and local $\mathrm{pH}^{15}$. Moreover, recent study by Ager et al. ${ }^{16}$ showed that different $\mathrm{C}_{2+}$ products are generated from specific active sites on $\mathrm{OD}-\mathrm{Cu}$ via isotope labeling. However, the current understanding of atom-scale active sites over OD-Cu falls short of expectation, impeding the rational design of catalysts converting $\mathrm{CO}_{2}$ to specific product, like ethylene and ethanol, with high selectivity. The difficulty is that available characterization techniques cannot detect the complex surface features of $\mathrm{OD}-\mathrm{Cu}$ due to the insufficient spatial resolution. Meanwhile, the computational demanding quantum mechanics (QM) level calculations are impractical for large-scale and long-term simulation, thus inhibiting the display of the "oxide-derived" process and further comprehension of atomic structures on authentic OD-Cu surface models. Therefore, identifying atom-scale product-specific active sites for $\mathrm{CO}_{2} \mathrm{R}$ over OD-Cu catalysts remains grand challenges.

The development of machine-learning-based new calculation methods is a frontier in the field of theoretical catalysis, at the same time, a promising route to analysis the active sites in complex heterogeneous catalytic system ${ }^{17,18}$. Goddard and coworkers ${ }^{19}$ computationally synthesized copper nanoparticles $(\mathrm{Cu}$ NP) by simulating the chemical vapor deposition (CVD) process and used machine-learning method to explore the active sites for $\mathrm{C}-\mathrm{C}$ coupling. However, it is unclear that the surface structure of $\mathrm{Cu} \mathrm{NP}$ can be compared to that of OD-Cu. OD-Cu differs from $\mathrm{Cu} \mathrm{NP}$ or poly-Cu in that it shows higher selectivity for $\mathrm{C}_{2+}$ oxygenates at lower overpotential ${ }^{9,10}$, which means that active sites for $\mathrm{C}_{2+}$ products on OD-Cu may be different from that on $\mathrm{Cu}$ NP. In addition, active sites for specific products should be further clarified. Thus, new calculation method should be developed to simulate the "oxide-derived" process to obtain the authentic OD-Cu surface model.

Here, we break the spatiotemporal limitation of QM and use molecular dynamic simulation with global neural network potential (NN-MD) ${ }^{20,21}$ to simulate the whole dynamic evolution of the surface from copper oxide to OD-Cu. By combining NN potential-based high-throughout testing, density functional theory calculations and experimental studies, we establish the linkage correlations between the atomic structure of active sites and their catalytic activities for specific products in $\mathrm{CO}_{2} \mathrm{R}$ over $\mathrm{OD}-\mathrm{Cu}$ catalysts. This work provides theoretical guidance for the design of highly selective Cu-based catalysts and develops a versatile research framework, machine-learning accelerated theoretical calculations, coupled with actionable experiments, for analyzing atomic structures of active sites in heterogeneous catalytic systems.

\section{Results}

A realistic surface model is the prerequisite for the exact analysis of atomic structure of active sites. We employed molecular dynamics simulation with neural network potential $(\mathrm{NN}-\mathrm{MD})^{20,21}$ to simulate the whole reduction process of the oxide precursor. Experimentally, $\mathrm{OD}-\mathrm{Cu}$ are mainly derived from $\mathrm{Cu}_{2} \mathrm{O}$ phase ${ }^{9,22}$, thus $\mathrm{Cu}_{2} \mathrm{O}$ (111), the most stable facet ${ }^{23}$, was chosen as the precursor for the reduction process. To simulate the "oxide-derived" process, the two-step approach was employed. First, NN-MD was performed with canonical ensemble (NVT) to obtain a thermodynamically balanced surface structure. Subsequently, the oxygen vacancy formation energies $\left(E_{\mathrm{ov}}\right)$ of surface $\mathrm{O}$ were calculated, wherein $\mathrm{O}$ atoms with lower vacancy formation energies were removed to represent the reduction process, thereby forming a partially reduced surface. Afterwards, another round NN-MD simulation was carried out followed by the reduction, i.e., removal of surface $\mathrm{O}$ atoms, until $\mathrm{O}$ can no longer migrate to the surface and all been trapped in the third layer or below (Fig. 1a and Supplementary Figs. 1-12). (for details, see the "Methods") Previous research has shown that O below the third layer had little effect on the adsorption behavior of adsorbates ${ }^{24}$, therefore we believe that our OD-Cu surface models are

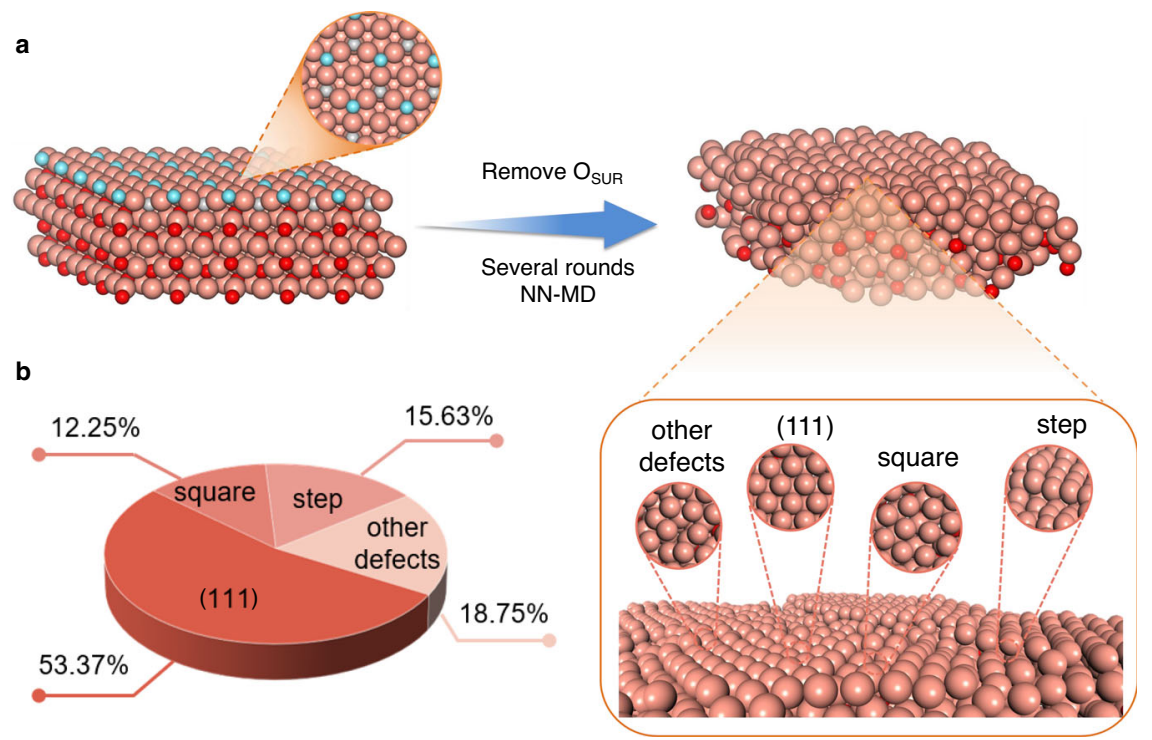

Fig. 1 Theoretical simulation of oxide-derived process. a Illustration of our procedure to construct OD-Cu model with molecular dynamic simulation. Color code: brown-Cu; blue-surface O; gray-subsurface O; red-bulk O. b Proportions of different surface structures. 
a

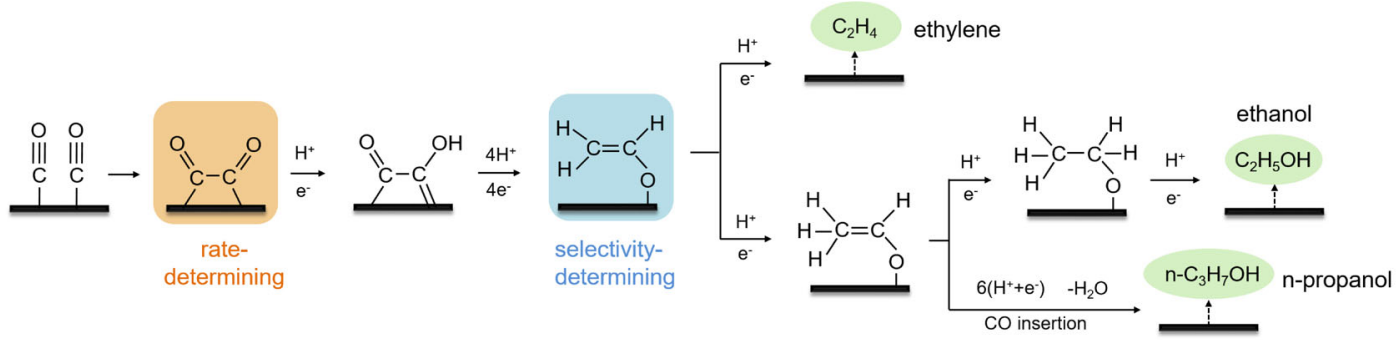

b

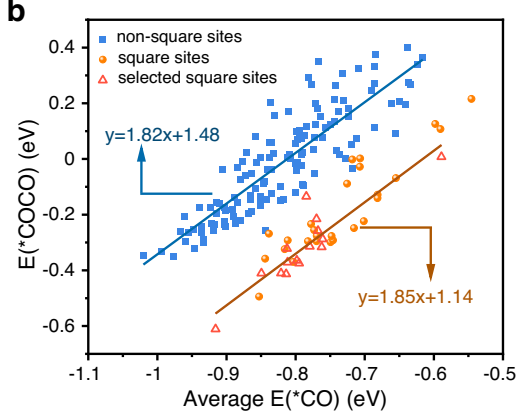

e

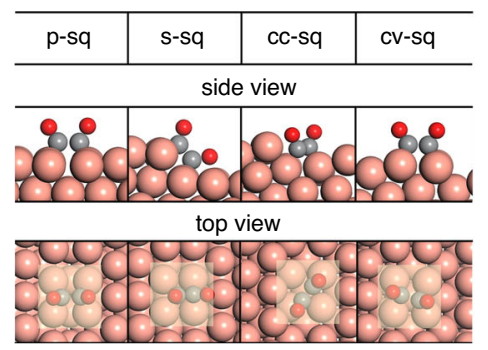

C

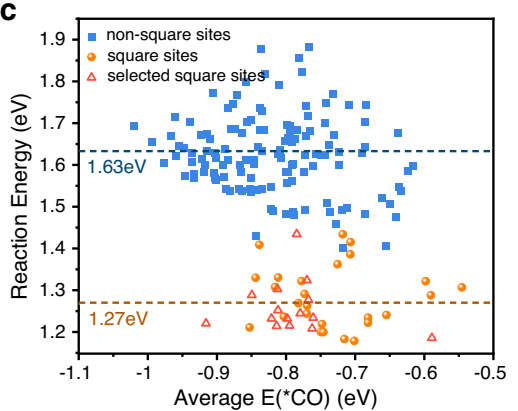

f

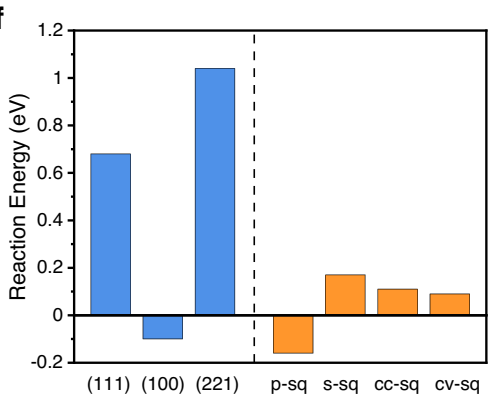

d

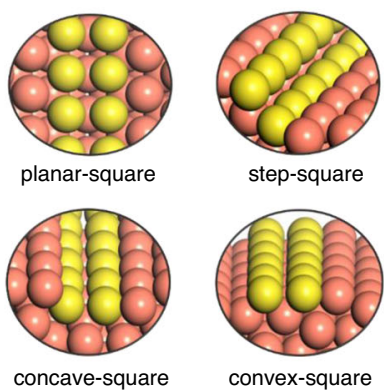

g

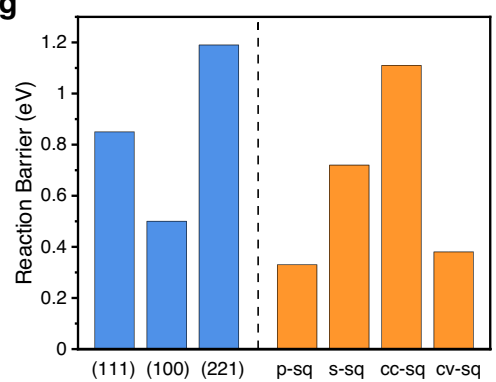

Fig. 2 Identification of $\mathbf{C}-\mathbf{C}$ coupling active sites. a Reaction pathway for $\mathrm{C}_{2+}$ products in $\mathrm{CO} 2 \mathrm{R}$. $\mathbf{b}$ The adsorption energy of ${ }^{\star} \mathrm{COCO}$ as the function of the average adsorption energy of two adsorbed ${ }^{\star} \mathrm{CO}$. $\mathbf{c}$ Reaction energy $\left(2{ }^{\star} \mathrm{CO} \rightarrow{ }^{\star} \mathrm{COCO}\right)$ as the function of the average $\mathrm{E}\left({ }^{\star} \mathrm{CO}\right)$ on these sites. $\mathbf{d} \mathrm{DFT}$ periodic slab models of four square-like sites. ${ }^{\star}{ }^{\star} \mathrm{OCCO}$ configurations on these four sites (solvent molecules have been removed to show the adsorbate configurations). f Reaction energies and $\mathbf{g}$ barriers $\left(2{ }^{\star} \mathrm{CO} \rightarrow{ }^{\star} \mathrm{COCO}\right)$ on different DFT slab models under appropriate electrochemical interface. Color code: brown-Cu; yellow-Cu in square-like sites; gray-C; red-O.

consistent with the current experimental research that metallic copper is the active phase for $\mathrm{CO}_{2} \mathrm{R}$ over OD-Cu rather than the oxide phase $\mathrm{e}^{25}$.

In our simulated OD-Cu surface structures, (111) facet dominates, occupying about 53\% surface area (Fig. 1b). Besides, there are some obvious steps, which resemble $\mathrm{Cu}(\mathrm{S})-[n(111) \times(111)]$ step sites, where one atomic height of (111) step is introduced into several atomic rows of (111) terrace. Moreover, a few defects like grain boundaries and vacancies exist (Supplementary Fig. 13). $\mathrm{Up}$ to now, authentic OD-Cu surface structures are obtained based on NN-MD simulation of the whole reduction process.

To improve the activity and selectivity for $\mathrm{C}_{2+}$ products of the catalyst, it is crucial to understand the nature of the active sites presented on $\mathrm{Cu}$ catalyst. The pathway for $\mathrm{C}_{2+}$ products ${ }^{26-28}$ is illustrated in Fig. 2a. Solid experimental researches show that $\mathrm{CO}_{2}$ electroreduction to multi-carbon products is independent on the standard hydrogen electrode (SHE) scale and $\mathrm{C}-\mathrm{C}$ coupling step that does not involve proton transfer is considered as ratedetermining step (RDS) ${ }^{29}$, which means the rate of $\mathrm{C}-\mathrm{C}$ coupling determines the whole activity for $\mathrm{C}_{2+}$ products over $\mathrm{OD}-\mathrm{Cu}$ catalysts. Both vibrational spectra ${ }^{30,31}$ and kinetic models ${ }^{32,33}$ reveal that ${ }^{*} \mathrm{CO}$ is the main intermediate on $\mathrm{Cu}$ surface under reduction conditions. Considering that $\mathrm{C}_{2}$ pathway displays a $\mathrm{pH}$ independent on SHE scale, $2^{*} \mathrm{CO}$ dimerization is thus the most accepted form for $\mathrm{C}-\mathrm{C}$ coupling on copper surface ${ }^{34}$.
We then randomly selected 155 surface sites on OD-Cu surface models to explore this dimerization process. As shown in Fig. 2b, some sites obviously show stronger ${ }^{*} \mathrm{COCO}$ binding energies. Interesting, these sites have the same feature that they all own the square-like orientation. We then selected another 15 square sites from OD-Cu surfaces to verify this tendency. Not surprisingly, these square sites can efficiently stabilize the *COCO intermediate as well. Two disparate scaling relationship can be obtained between the average adsorption energy of two binding * $\mathrm{CO}(E(\mathrm{CO}))$ and coupled intermediate *COCO $(E(\mathrm{COCO}))$ on the non-square sites and square sites. Moreover, these two relationships almost share the same slope but have a $0.34 \mathrm{eV}$ intercept difference. This value is very close to the adsorption energy difference, $0.31 \mathrm{eV}$, of ${ }^{*} \mathrm{COCO}$ over $\mathrm{Cu}(111)$ and $\mathrm{Cu}(100)$, indicating the decreased intercept is merely attributed to the additional stabilizing effect for ${ }^{*} \mathrm{COCO}$ intermediate at square-like sites. Reflected in reaction energy of $\mathrm{C}-\mathrm{C}$ coupling process (Fig. 2c), the average reaction energy on square sites is $1.27 \mathrm{eV}, 0.36 \mathrm{eV}$ lower than that on non-square sites. Therefore, square-like sites are the most potential candidates to serve as active sites for $\mathrm{C}-\mathrm{C}$ coupling.

Further analysis found that these square sites have different local structures. Therefore, we classified these sites into four types: planar-square (p-sq), step-square (s-sq), concave-square (cc-sq) and convex-square (cv-sq). p-sq sites are the $\Sigma 3$ grain boundary 
created by two flat (111) planes with $60^{\circ}$ lattice orientation difference. s-sq sites are similar to $\mathrm{Cu}(\mathrm{S})-[n(111) \times(100)]$ step sites where (100) steps are introduced to (111) basal plane. While cc-sq and $\mathrm{cV}$-sq are formed by rotation and then splicing of two (111) planes. In order to explore $\mathrm{C}-\mathrm{C}$ coupling more accurately, we abstracted these four surface sites into small slab models for accurate density functional theory (DFT) calculations (Fig. 2d), which are expected to have similar chemical properties to the selective sites from the MD models (Methods and Supplementary Tables 4-6). Here, (322) and (221) facet, 5(111) $\times(100)$ and 5 $(111) \times(111)$, represent $s$-sq sites and other step sites, respectively.

Our calculation shows that $\mathrm{CO}$ dimerization reaction over these four square-like structures are less endothermic than that over (111) and (221) facet (Fig. 2e, f) under proper electrochemical interface. Among them, p-sq exhibits the lowest reaction energy of $-0.16 \mathrm{eV}$. Moreover, we found that the reaction barriers of $\mathrm{C}-\mathrm{C}$ coupling on p-sq, s-sq and $\mathrm{cv}$-sq sites are $0.33 \mathrm{eV}$, $0.72 \mathrm{eV}$, and $0.39 \mathrm{eV}$, respectively, much lower than $0.85 \mathrm{eV}$ and $1.19 \mathrm{eV}$ on (111) and (221) facets (Fig. 2g). Based on the analysis above, three active sites, $\mathrm{p}$-sq, s-sq and cv-sq sites, which favor for $\mathrm{C}_{2+}$ products production have been identified on OD-Cu.

Previous work proved that ethylene and ethanol are not generated from the same types of active sites, which means the $\mathrm{C}-\mathrm{C}$ coupling active sites can be further subdivided ${ }^{16}$. From calculated free energy profiles of the whole reaction pathway (Supplementary Figs. 24-26), ${ }^{*} \mathrm{CH}_{2} \mathrm{CHO}$ serves as the selectivity determining intermediate (SDI), which corresponds to previous calculations on copper single crystal facets ${ }^{27,28}$. The ethylene pathway proceeds with cleavage of $\mathrm{C}-\mathrm{O}$ bond while alcohols are formed by further protonation. On p-sq and $\mathrm{cv}$-sq sites (Fig. 3a, b), the cleavage of $\mathrm{C}-\mathrm{O}$ bond to form $\mathrm{C}_{2} \mathrm{H}_{4}$ is thermodynamic favored compared to the protonation of a-C to form ${ }^{*} \mathrm{CH}_{3} \mathrm{CHO}$ intermediate. Conversely, s-sq site shows strong thermodynamic tendency for further hydrogenation, thus inhibiting the $\mathrm{C}_{2} \mathrm{H}_{4}$ pathway and meanwhile promoting alcohols production (Fig. $3 \mathrm{c}$ ).
These results suggest that p-sq and $\mathrm{cv}$-sq sites are responsible for generating ethylene while s-sq favors the alcohols production.

Interestingly, we found that the tendency to produce ethylene or alcohols can be simply predicted by the $\mathrm{C}-\mathrm{O}$ bond length of ${ }^{*} \mathrm{CH}_{2} \mathrm{CHO}$. The shortest $\mathrm{C}-\mathrm{O}$ bond length $(1.296 \AA)$ is in ${ }^{*} \mathrm{CH}_{2} \mathrm{CHO}$ on s-sq site, followed by that on p-sq and cv-sq sites ( $1.315 \AA$ and $1.322 \AA$ ). To further quantitatively evaluate the strength of the bond, we conducted the integrated crystal orbital Hamilton population (ICOHP) analysis of C-O bond (Fig. 3d). The shorter bond length and larger value of -ICOHP indicates that the $\mathrm{C}-\mathrm{O}$ bond is harder to break, which means the ethylene pathway is inhibited while alcohols pathway is favored. Indeed, the difference between the Gibbs-free reaction energy to break $\mathrm{C}-\mathrm{O}$ bond and further protonation is negatively correlated to the $\mathrm{C}-\mathrm{O}$ bond length (Fig. 3d). Remarkably, s-sq class, the $\mathrm{Cu}(211)$, $\mathrm{Cu}(533), \mathrm{Cu}(755)$ and $\mathrm{Cu}(433)[n=3,4,6,7$ in $n(111) \times(100)]$ all exhibit the same tendency like (322) facet. It is noteworthy, that, $\mathrm{Cu}(100)$ and $\mathrm{Cu}(751)$, which experimentally show more selective for ethylene and alcohols ${ }^{7}$, fall in their respective product region, thus meaning the bond length of $\mathrm{C}-\mathrm{O}$ in adsorbed ${ }^{*} \mathrm{CH}_{2} \mathrm{CHO}$ may be a simple descriptor for the selectivity of ethylene and alcohols on different surface structures.

The specific geometry of step-square site explains the difference in selectivity. Figure $3 e$ shows that the general coordination number $(\mathrm{GCN})$ of $\mathrm{s}$-sq is the lowest (5.50), thus showing the strongest adsorption energy of acetaldehyde intermediate $\left({ }^{*} \mathrm{CH}_{3} \mathrm{CHO}\right)$, leading to the hydrogenation pathway dominances. Meanwhile, p-sq and cv-sq can supply the fourfold sites to stabilize the newly formed ${ }^{*} \mathrm{O}$ after $\mathrm{C}-\mathrm{O}$ cleavage. While on s-sq site, ${ }^{*} \mathrm{O}$ can only adsorb in adjacent triple site, resulting in the instability of $* \mathrm{O}$, thereby further hindering the ethylene pathway (Fig. 3f). Based on the analysis above, we elucidated how the geometry of $\mathrm{C}-\mathrm{C}$ active sites affects the bifurcation pathway, resulting in the difference of the selectivity for $\mathrm{C}_{2+}$ products. Furthermore, we can propose that the key to generating ethanol is that square-like sites coupled with low coordinated sites, where
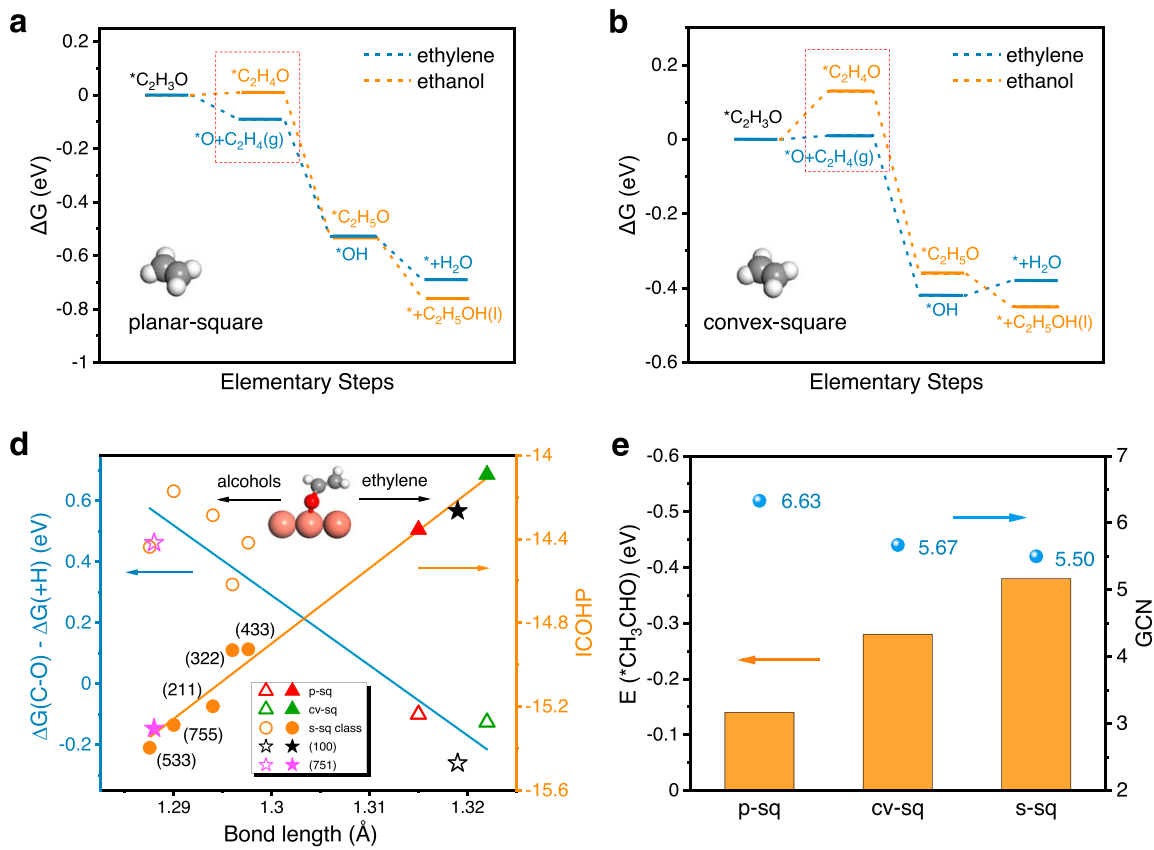
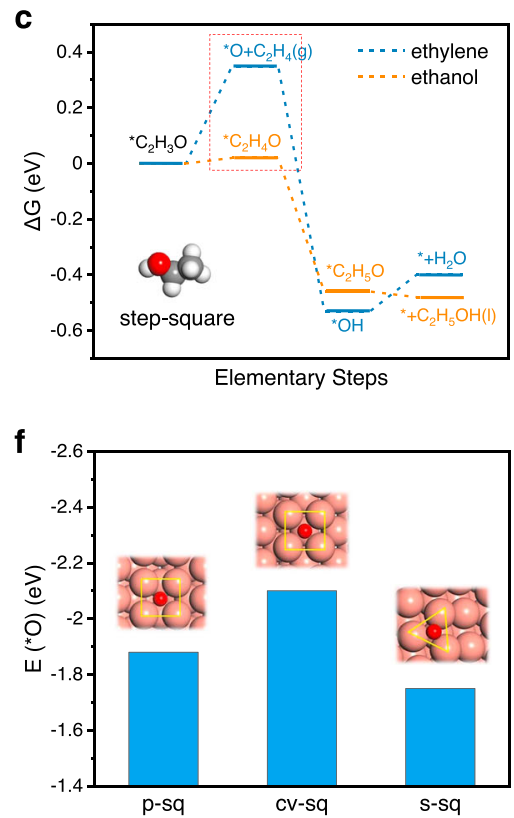

Fig. 3 Identification of product-specific active sites. Energy profile to form ethylene (blue line) and ethanol (orange line) for a planar-square site, b convex-square site, and c step-square site. d Blue: The relationship between the bond length of $\mathrm{C}-\mathrm{O}$ in adsorbed $\mathrm{CH}_{2} \mathrm{CHO}$ and the difference of $\mathrm{Gibbs}$-free reaction energy of $\mathrm{C}-\mathrm{O}$ scission $(\Delta \mathrm{G}(\mathrm{C}-\mathrm{O}))$ and hydrogenation $(\Delta \mathrm{G}(+\mathrm{H})$ ). Orange: The relationship between the bond length of $\mathrm{C}-\mathrm{O}$ in adsorbed $\mathrm{CH}_{2} \mathrm{CHO}$ and the ICOHP of $\mathrm{C}-\mathrm{O}$ bond. e General coordination number and adsorption energy of ${ }^{\star} \mathrm{CH} \mathrm{CHO} \mathrm{Cn}$ these three active sites. $\mathbf{f}$ Adsorption energy of ${ }^{\star} \mathrm{O}$ on these three active sites. 
a

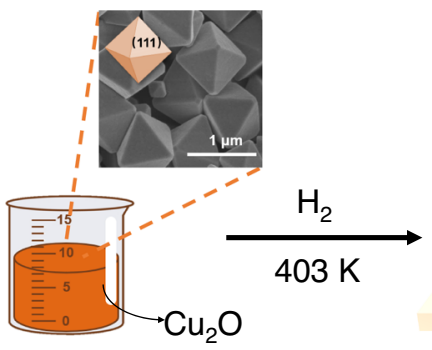

b

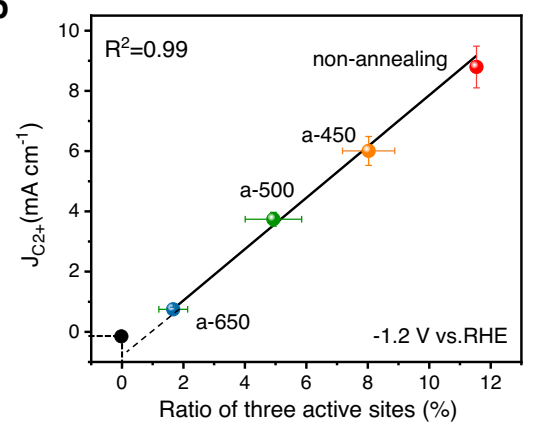

d

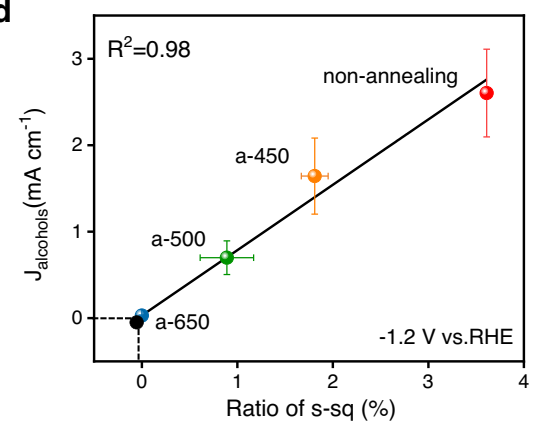

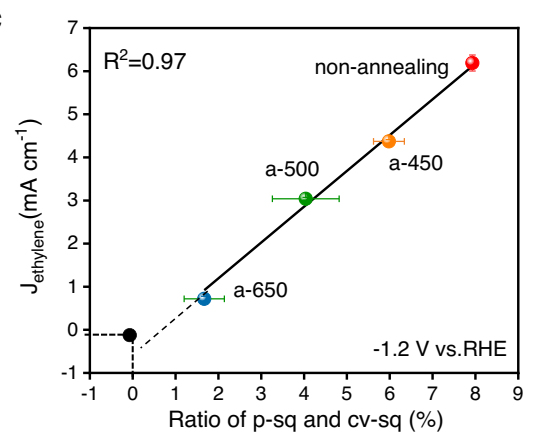

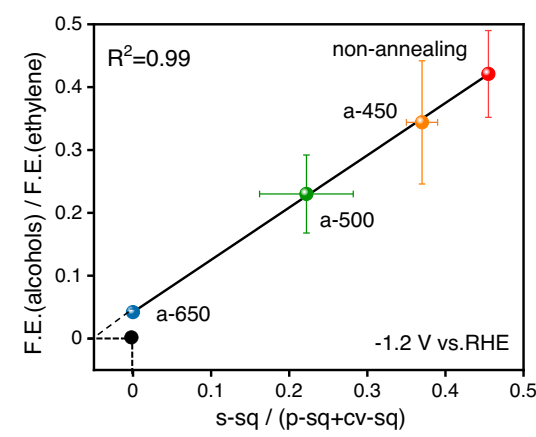

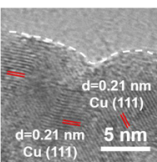
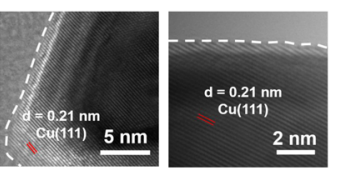

$450 \mathrm{~K}$

$500 \mathrm{~K}$

$650 \mathrm{~K}$ annealing

Increasing annealing $\mathrm{T}$

$\mathbf{f}$

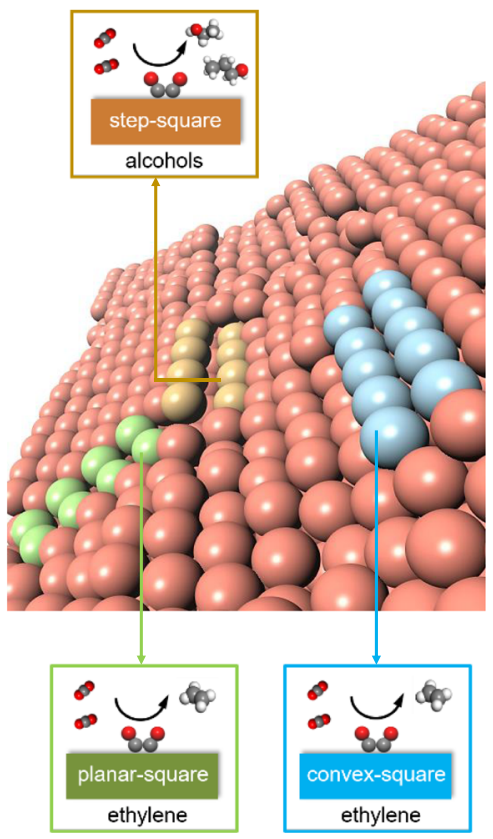

Fig. 4 Verification of active sites via thermal annealing process. a Schematic illustration of OD-Cu preparation and annealing process in experiment. The upper layer, TEM image of $\mathrm{Cu}_{2} \mathrm{O}$, SEM images of OD-Cu, a-450, a-500, and a-650 from left to right. The white dotted lines indicate the rough particle surface. The correlation between the current density of $\mathbf{b} \mathrm{C}_{2+}$ products and the ratios of all three active sites $\mathbf{c}$ ethylene and the ratios of planar-square and convex-square sites $\mathbf{d}$ alcohols and the ratios of step-square sites. e Alcohols/ethylene ratios as the function of the ratios of step-square sites to the sum of planar-square and convex-square sites at $-1.2 \mathrm{~V}$ vs. RHE. f Proposed structure-activity relationship diagram on OD-Cu in $\mathrm{CO}_{2} \mathrm{R}$. a- 450 , a- 500 , and a- 650 represent annealing at 450,500, and $650 \mathrm{~K}$, respectively. The error bars represent the standard deviation from three independent measurements.

square-like sites are responsible for $\mathrm{C}-\mathrm{C}$ coupling, low coordinated sites are conducive to further protonation for alcohols. Very recent study ${ }^{35}$ found that square, four-atom $\mathrm{Cu}$ islands are the active sites for generating ethanol. This structure certainly meets our design criteria for alcohols production. Although our OD-Cu models didn't directly observe such structure due to the scale limitation, their conclusion is consistent with ours.

To further illustrate the correlation between the catalytic activity for certain $\mathrm{C}_{2+}$ products on $\mathrm{OD}-\mathrm{Cu}$ and these active sites, we experimentally synthesized $\mathrm{OD}-\mathrm{Cu}$ catalysts, which are derived by $\mathrm{Cu}_{2} \mathrm{O}$ octahedron, where (111) facet dominates. Then, we varied defect concentrations by annealing the OD-Cu at 450, 500, and $650 \mathrm{~K}$ (Fig. 4a and Supplementary Figs. 14 and 15). Meanwhile, similar annealing operations were performed theoretically by NN-MD simulation to change the amount of the active sites, thus we can accurately count the ratios of active sites under different annealing temperature conditions from theoretical models (for details, see the "Methods"). After annealing, step sites and some defects are erased in this process and transform into flat (111) facet in general (Supplementary Figs. 16 and 17), which is consistent with previous work that the surface roughness decreased after annealing ${ }^{10}$ and our cyclic voltammetry (CV) curves results (Supplementary Fig. 18).
The ratio of three theoretically identified active sites, p-sq, s-sq, and $\mathrm{CV}$-sq sites, decreased with increasing annealing temperature as well. From our theoretical models, the OD-Cu surface before annealing has the largest proportion, $11.54 \%$, of these three active sites. The percentage drops to $9.37 \%, 4.93 \%$ and $1.67 \%$ after annealing at 450,500 , and $650 \mathrm{~K}$, respectively. When plotted against the catalytic activity, a linear relation is obtained between the surface area-normalized current density of $\mathrm{C}_{2+}$ products at $-1.2 \mathrm{~V}$ vs. RHE and the proportion of these three active sites (Fig. 4b). More specifically, the experimental current densities of ethylene and alcohols are linearly correlated to the density of sum of the density of p-sq and $\mathrm{cv}$-sq sites, and the density of s-sq sites, respectively (Fig. 4c, d). The linear correlations are also existed at -1.0 and $-1.1 \mathrm{~V}$ vs. RHE (Supplementary Figs. 21 and 22). Moreover, the ratio of catalytic activities of alcohols to ethylene is proportional to the ratio of the amount of s-sq to (p-sq $+\mathrm{cv}$-sq) (Fig. 4e). If we change the ordinate from F.E.(alcohols)/F.E.(ethylene) to F.E. (ethylene)/F.E.(alcohols), the positive correlation no long exists (Supplementary Fig. 23), which means the catalytic activities of alcohols and ethylene have no correlation with (p-sq $+\mathrm{cv}-\mathrm{sq})$ and $\mathrm{s}$-sq, respectively. This in turn suggests that the $\mathrm{CO}_{2}$ reduction activity for ethylene and alcohols strongly depends on their specific active sites. Notably, the intercepts of these relations are all close to 
0 , meaning that almost all the catalytic activity for these products arises from their specific active sites. Based on this quantitative structure-activity relationship, we can demonstrate that p-sq and $\mathrm{cv}$-sq sites are responsible for ethylene production while s-sq sites promote generating alcohols on OD-Cu surface (Fig. 4f). Therefore, the key to boosting the highly valuable $\mathrm{C}_{2+}$ alcohols production is to synthesize $\mathrm{Cu}$-based catalysts with more step-square sites by adjusting the precursors or the synthesis conditions.

Although the activities of ethylene and alcohols both showed a downward trend with the increase of annealing temperature, it can be clearly seen that the activity to produce alcohols decreases faster, resulting in the decreased selectivity of alcohols to ethylene with the increasing annealing temperature (Fig. 4e). The reason is that in the annealing process, the steps are easily erased, leading to the fast decrease of the s-sq coverage. However, the grain boundary like p-sq sites are stuck in two (111) planes, which are relatively robust, leading to the activity of ethylene decreases more slowly.

This work reveals the atomic-level product-specific active sites of $\mathrm{CO}_{2} \mathrm{R}$ over $\mathrm{OD}-\mathrm{Cu}$ catalysts by combining theoretical and experimental methods, where planar-square and convex-square sites are identified to be the active sites for ethylene production while step-square sites are responsible for the generation of $\mathrm{C}_{2+}$ alcohols. These new insights resolved the long-term debate over the active sites on OD-Cu catalysts and identified how each site works, which are the prerequisites for the development of highly selective, i.e., $\mathrm{C}_{2+}$ oxygenates, $\mathrm{Cu}$-based catalysts for $\mathrm{CO}_{2} \mathrm{R}$ in the future. Furthermore, this work also provides a versatile tool for the analysis of the complicated heterogeneous catalytic system.

\section{Methods}

Computational details. All simulations with neural network potential were carried out by LASP software (www.lasphub.com) ${ }^{20,21}$. Molecular dynamic simulation with neural network potential (NN-MD) was performed at a constant temperature $(\mathrm{T}=300 \mathrm{~K})$ within the NVT ensemble. Details in neural network potential are seen in Supplementary Methods.

Simulation of the oxide-derived process: We started this process with pristine $\mathrm{Cu}_{2} \mathrm{O}$ (111). Previous studies showed that surface $\mathrm{O}$ are unstable on $\mathrm{Cu}_{2} \mathrm{O}$ at -0.1 $\mathrm{V}$ (vs. RHE) ${ }^{36}$. Therefore, all the surface $\mathrm{O}$ atoms in our slab model, which share the same environment, were removed without distinction. Then, we carried out 1 ns NN-MD at $300 \mathrm{~K}$ to obtain the first-stage in the reduction process. After $1 \mathrm{~ns}$ simulation, the surface reconstruction was severe and the morphology became chaotic. Some near-surface bulk O migrated to the surface. At this time, the chemical environments of surface $\mathrm{O}$ were diverse. It is unreasonable to remove all the surface $\mathrm{O}$ at this time simultaneously. Thus, we consider the $E_{\mathrm{ov}}$ as the criterion to remove surface $\mathrm{O}$. The $E_{\mathrm{ov}}$ of all the surface oxygen has been calculated by NN potential (The NN potential calculated $E_{\text {ov }}$ are shown in Supplementary Table 3, leading to RMSE (the root mean squared error) $=0.12 \mathrm{eV}$ ). In general, we found that 3-coordinated (3-coor) oxygen correspond to the lower oxygen vacancy formation energy, which is consistent with the instability of 3-coor $\mathrm{O}$ on the perfect $\mathrm{Cu}_{2} \mathrm{O}$ surface ${ }^{23}$. Therefore, when removing surface $\mathrm{O}$, coordination number is used as the first criterion and the $E_{\mathrm{ov}}$ as the second. The one kind is that all 3-coor surface $\mathrm{O}$ are removed. The another is that 4 -coor $\mathrm{O}$ whose $E_{\mathrm{ov}}$ locates in the range of $E_{\text {ov }}$ of 3-coordinated $\mathrm{O}$ are removed together with 3-coor $\mathrm{O}$. Through this standard, two types of second-stage structures were obtained. Subsequently, $1 \mathrm{~ns}$ NN-MD simulation was performed on these two structures to approach equilibrium. Next step for removing $\mathrm{O}$ was the same as above until no oxygen can move to the surface. All surface structures are shown in Supplementary Fig. 13, which are marked with OD-Cu-M(M = 1-6). It was worth noting that in the process of NN-MD simulation, some structures have collapsed and cannot maintain the structure of slab owing to the periodicity of slab models, which were not considered in our work.

The calculation of adsorption energy of * $\mathrm{CO}$ and * COCO on OD-Cu surface are conducted by neural network potential as well. In order to verify the accuracy of this NN potential, we randomly selected 50 and 15 surface sites on OD-Cu-1 for costly DFT single point calculation for $\mathrm{E}\left({ }^{*} \mathrm{CO}\right)$ and $\mathrm{E}\left({ }^{*} \mathrm{COCO}\right)$. The root mean square error (RMSE) of ${ }^{*} \mathrm{CO}$ and ${ }^{*} \mathrm{COCO}$ adsorption energy between the NN prediction and DFT calculation is as low as $0.08 \mathrm{eV}$ and $0.13 \mathrm{eV}$, respectively. (Supplementary Tables 4 and 5)

The process of annealing at $400 \mathrm{~K}: \mathrm{OD}-\mathrm{Cu}$ is simulated at $330,360,400$, and $450 \mathrm{~K}$ for $0.2,0.2,0.2$, and $0.6 \mathrm{~ns}$ in order, to simulate the process of heating process, and then simulated at $400,360,330$, and $300 \mathrm{~K}$ for $0.2 \mathrm{~ns}$, respectively, in order, to simulate the process of cooling. The process of annealing at $500 \mathrm{~K}$ : OD-Cu is simulated at 360,420 , and $500 \mathrm{~K}$ for $0.2,0.2$, and $0.6 \mathrm{~ns}$ in order, to simulate the process of heating process, and then simulated at 420,360 , and $300 \mathrm{~K}$ for $0.2 \mathrm{~ns}$, respectively in order, to simulate the process of cooling. The process of annealing at $650 \mathrm{~K}: \mathrm{OD}-\mathrm{Cu}$ is simulated at $400,500,600$, and $650 \mathrm{~K}$ in order of $0.2,0.2,0.2$, and $0.6 \mathrm{~ns}$ to simulate the process of heating, and then at $600,500,450$, and $300 \mathrm{~K}$ in order, to simulate the process of cooling at $0.2 \mathrm{~ns}$.

In our article, we performed $0.2 \mathrm{~ns}$ NN-MD in each heating and cooling stage. We then changed the residence time $(0.1 \mathrm{~ns}$ and $0.3 \mathrm{~ns})$ at different annealing temperatures. As a result, we found that the percentages of each structural motifs remain basically unchanged at different annealing temperature (Supplementary Table 10), which means, at least for NN-MD, the annealing time does not change the trend of the ratio of active sites. In all, $0.2 \mathrm{~ns}$ is enough for obtaining the ratio of each structural motifs considering the computational cost.

Density functional theory calculations were performed using the plane-wavebased Vienna Ab Initio Simulation package (VASP) ${ }^{37}$. The electron exchange and correlation effects were described by the generalized gradient approximation (GGA) in the form of the Perdew-Burke-Ernzerhof (PBE) functional ${ }^{38}$. The D3 correction method was employed to illustrate the long-range dispersion interactions between the adsorbates, water and $\mathrm{Cu}$ surface ${ }^{39}$. The interaction between atomic cores and electrons was described by the projector augmented wave (PAW) method ${ }^{38}$. A cutoff energy of $400 \mathrm{eV}$ for the plane-wave basis set and an atomic force convergence of $0.02 \mathrm{eV} / \AA$ were employed. The transition state search was conducted with climbing image nudged elastic band (CI-NEB) method $^{40}$, followed by the dimer method to converge the saddle point within $0.05 \mathrm{eV} / \AA$.

When theoretically investigating electrochemical barriers, it is essential to model suitable electrochemical interface. Previous study has found that one layer of water can accurately describe the adsorption behavior compared to more water layers and a simple hydronium ion can induce the same electric field as cation does $^{41-43}$. Thus, we established one layer of water with an added hydronium ion to simulate the electrochemical interface.

(111), (100), and (221) facets were modeled using $(3 \times 3),(3 \times 3)$, and $(1 \times 3)$ supercells with $(3 \times 3 \times 1) \mathrm{k}$-point grid. $(1 \times 3)$ supercells were used to model stepsquare sites $(n(111) \times(100)):(211),(533),(322),(755)$ and $(433)$ facets, $(3 \times 3 \times 1)$, $(3 \times 3 \times 1),(3 \times 3 \times 1),(2 \times 3 \times 1)$ and $(2 \times 3 \times 1)$ k-point grid were used. $(2 \times 1)$ supercell was used to model $(751)$ facet and $(2 \times 3 \times 1)$ k-point grid were used. Four layers were used for above models, where the two bottom layers were kept fixed in optimization. Planar-square is the grain boundary created by two flat (111) planes with different lattice orientations. $(1 \times 3 \times 1)$ k-point grid was used. Concavesquare and convex-square are obtained by rotation and then splicing of two (111) planes. $(3 \times 3 \times 1) \mathrm{k}$-point grid was used for both.

Materials. $\mathrm{KHCO}_{3}, \mathrm{NaOH}$, ethanol, and isopropanol were all purchased from Tianjin Kemiou Chemical Reagent Co., Ltd. Polyvinylpyrrolidone (PVP, Mw = 15000, K15) was purchased from Tokyo Chemical Industry Co., Ltd. L-ascorbic acid was purchased from Sinopharm Chemical Reagent Co., Ltd. All reagents were used without any purification process. Ultra purity water $(18.25 \mathrm{M} \Omega \mathrm{cm})$ supplied by a UP Water Purification System was used in the whole experimental processes. $\mathrm{CO}_{2}, \mathrm{Ar}, \mathrm{H}_{2}, \mathrm{~N}_{2}$ all were supplied by Air Liquid ( $\left.\geq 99.999 \%\right)$.

Preparation of $\mathrm{Cu}_{2} \mathrm{O}$ octahedron. The synthesis of $\mathrm{Cu}_{2} \mathrm{O}$ octahedron nanoparticle by a facile method has been described previously ${ }^{44}$. In a typical synthesis, $1.5 \mathrm{~g}$ PVP was dissolved into an aqueous solution of $0.01 \mathrm{M} \mathrm{CuCl}_{2} \cdot 2 \mathrm{H}_{2} \mathrm{O}(100 \mathrm{~mL})$ under constant stirring and heated in $55^{\circ} \mathrm{C}$ water bath. Then $2.0 \mathrm{M} \mathrm{NaOH}$ aqueous solution $(10 \mathrm{~mL})$ was added dropwise into the above transparent light green solution. After stirring for $0.5 \mathrm{~h}, 0.6 \mathrm{M}$ ascorbic acid solution $(10 \mathrm{~mL})$ was added dropwise. The color of the solution gradually changed to turbid red. The mixed solution was kept under constant stirring and in $55^{\circ} \mathrm{C}$ water bath for $3 \mathrm{~h}$. Then the resulting precipitate was collected by centrifugation and washed with distilled water and ethanol over three times to remove the residual inorganic ions and polymer. Finally, the powder was dried in vacuum at $55^{\circ} \mathrm{C}$ for $5 \mathrm{~h}$.

Preparation of OD-Cu. The prepared $\mathrm{Cu}_{2} \mathrm{O}$ octahedron was placed in a tube furnace (Hefei KeJing Materials Technology Co., LTD) with flowing $\mathrm{H}_{2}$ at $200 \mathrm{sccm}$ and heated at $130^{\circ} \mathrm{C}$ for $2 \mathrm{~h}$. The reduced sample was cooled slowly to room temperature in $\mathrm{H}_{2}$

Preparation of a-450, a-500, and a-650. The OD-Cu powder was placed in a tube furnace in $200 \mathrm{sccm}$ flowing $\mathrm{N}_{2}$ and heated at 450,500 , and $650 \mathrm{~K}$ for $2 \mathrm{~h}$, respectively. The annealed electrodes were cooled slowly to room temperature in $\mathrm{N}_{2}$.

Characterization. Field-emission scanning electron microscopy (FESEM) (Hitachi $\mathrm{S}-4800,3 \mathrm{kV}$ ) was used to characterize the morphology and microstructure of the samples. Transmission Electron Microscopy (TEM), High-resolution TEM (HRTEM) images were obtained at $200 \mathrm{kV}$ (JEOL JEM-2100F).

Working electrode preparation. In all, $10 \mathrm{mg}$ of the catalyst was dispersed in $1 \mathrm{~mL}$ of isopropanol, including $20 \mu \mathrm{L}$ of Nafion solution ( $5 \mathrm{wt} \%$ ) by sonicating for $1 \mathrm{~h}$. 
Twenty microliters of the obtained solution was then loaded on a glassy carbon electrode (Gaossunion). The geometric surface area was $0.5027 \mathrm{~cm}^{2}$. The electrode was dried in ambient air for the subsequent $\mathrm{CO}_{2}$ electroreduction test.

Electrochemical measurement. Electrochemical tests were performed in a twocompartment H-cell (Supplementary Fig. 27). An anion exchange membrane (FAA-3-PK-130, Fumatech) was used. The electrolyte was $30 \mathrm{~mL}$ of $0.1 \mathrm{M} \mathrm{KHCO}_{3}$ solution saturated with $\mathrm{CO}_{2}$ gas in the cathode part for at least $30 \mathrm{~min}$ prior to the $\mathrm{CO}_{2}$ reduction test. During electrolysis, $\mathrm{CO}_{2}$ was constantly bubbled through the electrolyte at a flow rate of $10 \mathrm{sccm}$ to prevent depletion of $\mathrm{CO}_{2}$ in the electrolyte and to allow continuous analysis of gaseous products via a gas chromatograph. The flow rate of $\mathrm{CO}_{2}$ was controlled with a mass flow controller (Alicat Scientific). Titanium mesh coated with iridium was used as the counter electrode and $\mathrm{Ag} / \mathrm{AgCl}$ as the reference electrode (saturated $\mathrm{KCl}$, Gaossunion). The glassy carbon electrode loaded with the catalyst served as the working electrode. An electrochemical workstation (IVIUM CompactStat.e20250) was used to provide the external bias. The gas products were detected using an in-line multiple-gas analyser gas chromatography system (GC2060, Shanghai Ruimin Instrument Co., Ltd.). The liquid products were collected from the cathode and anode chambers after electrolysis and analyzed by headspace-gas chromatography (HS-GC). Applied cathode potentials after $i R_{\text {cell }}$ compensation (i is the applied current and $R_{\text {cell }}$ is the cell resistance) were converted to the RHE reference scale using $E_{\mathrm{RHE}}=E_{\mathrm{Ag} / \mathrm{AgCl}}+$ $0.197 \mathrm{~V}+0.059 \times \mathrm{pH} ; R_{\text {cell }}$ is determined by performing an electrochemical impedance spectroscopy measurement.

OH adsorption. In order to clarify the change in surface structures, we have investigated the electrosorption of oxygenated species (e.g., $\mathrm{O}$ and/or $\mathrm{OH}$ ) on the resulting OD-Cu particles surfaces to identify the exposed facets. The cyclic voltammograms were recorded using a customized $\mathrm{H}$-type electrochemical cell and a potentiostat (Autolab PGSTAT 204, Metrohm). A Hg/HgO electrode (with saturated $\mathrm{KCl}$ solution as the filling solution, GaossUnion) and a graphite rod (GaossUnion) were used as the reference and counter electrode, respectively. A solution of $1 \mathrm{M} \mathrm{KOH}$ was used as the electrolyte. Ar was delivered to the cathode compartment at a constant rate of $20 \mathrm{sccm}$. The cathode and anode compartments were separated with an anion exchange membrane (Fumasep, FAA-3-PK-130). The low-index facets for $f_{c c} \mathrm{Cu}$ crystals were labeled by comparing the CVs to those of single crystals shown in previous works ${ }^{45,46}$.

\section{Data availability}

The data supporting the findings of the study are available within the paper and its Supplementary Information.

\section{Code availability}

The software code for LASP and the NN potentials used within the article are available from the website http://www.lasphub.com.

Received: 5 August 2020; Accepted: 14 December 2020; Published online: 15 January 2021

\section{References}

1. De Luna, P. et al. What would it take for renewably powered electrosynthesis to displace petrochemical processes? Science 364, eaav3506 (2019).

2. Gao, D., Arán-Ais, R. M., Jeon, H. S. \& Roldan Cuenya, B. Rational catalyst and electrolyte design for $\mathrm{CO}_{2}$ electroreduction towards multicarbon products. Nat. Catal. 2, 198-210 (2019).

3. Birdja, Y. Y. et al. Advances and challenges in understanding the electrocatalytic conversion of carbon dioxide to fuels. Nat. Energy 4, 732-745 (2019).

4. Nitopi, S. et al. Progress and perspectives of electrochemical $\mathrm{CO}_{2}$ reduction on copper in aqueous electrolyte. Chem. Rev. 119, 7610-7672 (2019).

5. Pang, Y. et al. Efficient electrocatalytic conversion of carbon monoxide to propanol using fragmented copper. Nat. Catal. 2, 251-258 (2019).

6. Li, J. et al. Copper adparticle enabled selective electrosynthesis of $\mathrm{n}$-propanol. Nat. Commun. 9, 4614 (2018).

7. Hahn, C. et al. Engineering $\mathrm{Cu}$ surfaces for the electrocatalytic conversion of $\mathrm{CO}_{2}$ : Controlling selectivity toward oxygenates and hydrocarbons. Proc. Natl Acad. Sci. USA 114, 5918-5923 (2017).

8. Thang Dinh, C. et al. $\mathrm{CO}_{2}$ electroreduction to ethylene via hydroxidemediated copper catalysis at an abrupt interface. Science 360, 783-787 (2018).

9. Li, C. W., Ciston, J. \& Kanan, M. W. Electroreduction of carbon monoxide to liquid fuel on oxide-derived nanocrystalline copper. Nature 508, 504-507 (2014).

10. Verdaguer-Casadevall, A. et al. Probing the active surface sites for $\mathrm{CO}$ reduction on oxide-derived copper electrocatalysts. J. Am. Chem. Soc. 137, 9808-9811 (2015).
11. Lum, Y. et al. Coupling on oxide-derived copper catalysts for electrochemical $\mathrm{CO}_{2}$ reduction. J. Phys. Chem. C. 121, 14191-14203 (2017).

12. Eilert, A. et al. Subsurface oxygen in oxide-derived copper electrocatalysts for carbon dioxide reduction. J. Phys. Chem. Lett. 8, 285-290 (2017).

13. Feng, X., Jiang, K., Fan, S. \& Kanan, M. W. A direct grain-boundary-activity correlation for $\mathrm{CO}$ electroreduction on $\mathrm{Cu}$ nanoparticles. ACS Cent. Sci. 2, 169-174 (2016).

14. Jiang, $\mathrm{K}$. et al. Metal ion cycling of $\mathrm{Cu}$ foil for selective $\mathrm{C}-\mathrm{C}$ coupling in electrochemical $\mathrm{CO}_{2}$ reduction. Nat. Catal. 1, 111-119 (2018).

15. Ma, M., Djanashvili, K. \& Smith, W. A. Controllable hydrocarbon formation from the electrochemical reduction of $\mathrm{CO}_{2}$ over $\mathrm{Cu}$ nanowire arrays. Angew. Chem. Int. Ed. 55, 6680-6684 (2016).

16. Lum, Y. \& Ager, J. W. Evidence for product specific sites on oxide-derived $\mathrm{Cu}$ catalysts for electrochemical $\mathrm{CO}_{2}$ reduction. Nat. Catal. 2, 86-93 (2019).

17. Cheng, T., Xiao, H. \& Goddard, W. A. Nature of the active sites for CO reduction on copper nanoparticles; suggestions for optimizing performance. J. Am. Chem. Soc. 139, 11642-11645 (2017).

18. Sun, G. \& Sautet, P. Metastable structures in cluster catalysis from firstprinciples: structural ensemble in reaction conditions and metastability triggered reactivity. J. Am. Chem. Soc. 140, 2812-2820 (2018).

19. Huang, Y., Chen, Y., Cheng, T., Wang, L.-W. \& Goddard, W. A. Identification of the selective sites for electrochemical reduction of $\mathrm{CO}$ to $\mathrm{C} 2+$ products on copper nanoparticles by combining reactive force fields, density functional theory, and machine learning. ACS Energy Lett. 3, 2983-2988 (2018).

20. Huang, S. D., Shang, C., Kang, P. L., Zhang, X. J. \& Liu, Z. P. LASP: Fast global potential energy surface exploration. Wiley Interdiscip. Rev.: Comput. Mol. Sci. 9, e1415 (2019).

21. Huang, S.-D., Shang, C., Zhang, X.-J. \& Liu, Z.-P. Material discovery by combining stochastic surface walking global optimization with a neural network. Chem. Sci. 8, 6327-6337 (2017).

22. Li, C. W. \& Kanan, M. W. $\mathrm{CO}_{2}$ reduction at low overpotential on $\mathrm{Cu}$ electrodes resulting from the reduction of thick $\mathrm{Cu}_{2} \mathrm{O}$ films. J. Am. Chem. Soc. 134, 7231-7234 (2012).

23. Yang, P. et al. The functionality of surface hydroxy groups on the selectivity and activity of carbon dioxide reduction over cuprous oxide in aqueous solutions. Angew. Chem. Int. Ed. 57, 7724-7728 (2018).

24. Fields, M., Hong, X., Nørskov, J. K. \& Chan, K. Role of subsurface oxygen on $\mathrm{Cu}$ surfaces for $\mathrm{CO}_{2}$ electrochemical reduction. J. Phys. Chem. C. 122, 16209-16215 (2018).

25. Lum, Y. \& Ager, J. W. Stability of residual oxides in oxide-derived copper catalysts for electrochemical $\mathrm{CO}_{2}$ reduction investigated with (18) O labeling. Angew. Chem. Int. Ed. 57, 551-554 (2018).

26. Perez-Gallent, E., Figueiredo, M. C., Calle-Vallejo, F. \& Koper, M. T. Spectroscopic observation of a hydrogenated $\mathrm{CO}$ dimer intermediate during $\mathrm{CO}$ reduction on $\mathrm{Cu}(100)$ electrodes. Angew. Chem. Int. Ed. 56, 3621-3624 (2017).

27. Calle-Vallejo, F. \& Koper, M. T. Theoretical considerations on the electroreduction of $\mathrm{CO}$ to $\mathrm{C} 2$ species on $\mathrm{Cu}(100)$ electrodes. Angew. Chem. Int. Ed. 52, 7282-7285 (2013).

28. Zheng, Y. et al. Understanding the roadmap for electrochemical reduction of $\mathrm{CO}_{2}$ to multi-carbon oxygenates and hydrocarbons on copper-based catalysts. J. Am. Chem. Soc. 141, 7646-7659 (2019).

29. Wang, L. et al. Electrochemical carbon monoxide reduction on polycrystalline copper: effects of potential, pressure, and $\mathrm{pH}$ on selectivity toward multicarbon and oxygenated products. ACS Catal. 8, 7445-7454 (2018).

30. Wuttig, A. et al. Tracking a common surface-bound intermediate during $\mathrm{CO} 2$ to-fuels catalysis. ACS Cent. Sci. 2, 522-528 (2016).

31. Gunathunge, C. M., Ovalle, V. J., Li, Y., Janik, M. J. \& Waegele, M. M. Existence of an electrochemically Inert $\mathrm{CO}$ population on $\mathrm{Cu}$ electrodes in alkaline pH. ACS Catal. 8, 7507-7516 (2018).

32. Liu, X. et al. Understanding trends in electrochemical carbon dioxide reduction rates. Nat. Commun. 8, 15438 (2017).

33. Goodpaster, J. D., Bell, A. T. \& Head-Gordon, M. Identification of possible pathways for C-C bond formation during electrochemical reduction of $\mathrm{CO}_{2}$ : new theoretical insights from an improved electrochemical model. J. Phys. Chem. Lett. 7, 1471-1477 (2016).

34. Schouten, K. J., Qin, Z., Perez Gallent, E. \& Koper, M. T. Two pathways for the formation of ethylene in $\mathrm{CO}$ reduction on single-crystal copper electrodes. J. Am. Chem. Soc. 134, 9864-9867 (2012).

35. Pique, O., Vines, F., Illas, F. \& Calle-Vallejo, F. Elucidating the structure of ethanol-producing active sites at oxide-derived $\mathrm{Cu}$ electrocatalysts. ACS Catal. 10, 10488-10494 (2020)

36. Nie, X., Griffin, G. L., Janik, M. J. \& Asthagiri, A. Surface phases of $\mathrm{Cu}_{2} \mathrm{O}(111)$ under $\mathrm{CO}_{2}$ electrochemical reduction conditions. Catal. Commun. 52, 88-91 (2014).

37. Perdew, J. P., BurkeK. \& Ernzerhof, M. Generalized gradient approximation made simple. Phys. Rev. Lett. 77, 3865-3868 (1996).

38. Kresse, G. \& Joubert, D. From ultrasoft pseudopotentials to the projector augmented-wave method. Phys. Rev. B 59, 1758-1775 (1999). 
39. Grimme, S., Antony, J., Ehrlich, S. \& Krieg, H. A consistent and accurate ab initio parametrization of density functional dispersion correction (DFT-D) for the 94 elements H-Pu. J. Chem. Phys. 132, 154104 (2010).

40. Henkelman, G., Uberuaga, B. P. \& Jónsson, H. A climbing image nudged elastic band method for finding saddle points and minimum energy paths. $J$. Chem. Phys. 113, 9901-9904 (2000).

41. Gauthier, J. A., Dickens, C. F., Chen, L. D., Doyle, A. D. \& Nørskov, J. K. Solvation effects for oxygen evolution reaction catalysis on $\mathrm{IrO}_{2}(110)$. J. Phys. Chem. C. 121, 11455-11463 (2017).

42. Montoya, J. H., Shi, C., Chan, K. \& Norskov, J. K. Theoretical insights into a $\mathrm{CO}$ dimerization mechanism in $\mathrm{CO}_{2}$ electroreduction. J. Phys. Chem. Lett. 6, 2032-2037 (2015).

43. Resasco, J. et al. Promoter effects of alkali metal cations on the electrochemical reduction of carbon dioxide. J. Am. Chem. Soc. 139, 11277-11287 (2017)

44. Zhang, D.-F. et al. Delicate control of crystallographic facet-oriented $\mathrm{Cu}_{2} \mathrm{O}$ nanocrystals and the correlated adsorption ability. J. Mater. Chem. 19, 5220-5225 (2009).

45. Cao, L. et al. Mechanistic insights for low-overpotential electroreduction of $\mathrm{CO}_{2}$ to $\mathrm{CO}$ on copper nanowires. ACS Catal. 7, 8578-8587 (2017).

46. Luc, W. et al. Two-dimensional copper nanosheets for electrochemical reduction of carbon monoxide to acetate. Nat. Catal. 2, 423-430 (2019).

\section{Acknowledgements}

We thank Prof. Dr. Felix Studt for fruitful discussions. We acknowledge the National Key Research and Development Program of China (2016YFB0600901), the National Science Foundation of China $(21525626,21761132023)$ and the Program of Introducing Talents of Discipline to Universities (BP0618007) for financial support. We also acknowledge Tianjin University High-Performance Computing Center for calculation support.

\section{Author contributions}

J.G. conceived and coordinated the research. D.C., Z.Z., and P.Y. conducted molecule dynamics simulation and DFT calculations. Z.L. fitted the NN potential. G.Z. and H.G. contributed to catalyst synthesis and catalytic experiments. J.G., D.C., Z.Z., G.Z., L.L., S.L., X.C., S.C., T.W., and G.O. contributed analysis of data and discussion. D.C., Z.Z., and J.G. participated in writing the manuscript. All the authors participated in discussions of the research.

\section{Competing interests}

The authors declare no competing interests.

\section{Additional information}

Supplementary information is available for this paper at https://doi.org/10.1038/s41467020-20615-0.

Correspondence and requests for materials should be addressed to J.G.

Peer review information Nature Communications thanks Joel Ager III, and the other, anonymous, reviewer(s) for their contribution to the peer review of this work.

Reprints and permission information is available at http://www.nature.com/reprints

Publisher's note Springer Nature remains neutral with regard to jurisdictional claims in published maps and institutional affiliations.

(c) (i) Open Access This article is licensed under a Creative Commons Attribution 4.0 International License, which permits use, sharing, adaptation, distribution and reproduction in any medium or format, as long as you give appropriate credit to the original author(s) and the source, provide a link to the Creative Commons license, and indicate if changes were made. The images or other third party material in this article are included in the article's Creative Commons license, unless indicated otherwise in a credit line to the material. If material is not included in the article's Creative Commons license and your intended use is not permitted by statutory regulation or exceeds the permitted use, you will need to obtain permission directly from the copyright holder. To view a copy of this license, visit http://creativecommons.org/ licenses/by/4.0/.

(C) The Author(s) 2021 